\title{
OBSERVATIONS OF CIRCULAR POLARIZATION \\ IN V 1357 CYG =CYG X-1
}

\author{
O. S. SHULOV \\ Astronomical Observatory of Leningrad State University, U.S.S.R.
}

\begin{abstract}
Our observations of the X-ray binary V 1357 Cyg suggest that a small circular polarization increasing towards the longer wavelength exists in the light of the star.
\end{abstract}

If a compact object in an X-ray binary system has a large magnetic field, its radiation may well be circularly polarized. Unfortunately, the visible radiation of the object is extremely weak in comparison with that of the normal star in the binary. We cannot, therefore, expect a large circular polarization in the light of the system, but we need not exclude the possibility of detecting a small polarization near the accuracy limit of observation.

The ultraviolet and blue-green circular polarization has been proved by Gehrels (1972) to be less than $0.1 \%$ in V 1357 Cyg, a binary system identified with the X-ray source Cyg X-1. In June-November 1973 we carried out about a hundred observations of the circular polarization of the star in six wavelength passbands. The results were published by Shulov and Kopatskaya (1974) and can be summarized as follows.

(1) An upper limit to the variation in circular polarization is less then $0.1 \%$ in each passband over the time scale from a day to half a year.

(2) Table I shows the circular polarization averaged over the whole time interval mentioned above. The polarization does not exceed the 2- $\sigma$ level in all of the passbands but $\lambda 6300 \AA$ and agrees well with the result reported by Gehrels (1972). Nevertheless, there is a marked tendency in the measured polarization to rise with increasing wavelength. It does not appear to be by chance. We don't exclude a systematic error of unknown origin as a source of the tendency, but this seems to be unlikely.

TABLE I

Broad-band circular polarization measurements of V 1357 Cyg

\begin{tabular}{llllr}
\hline $\begin{array}{l}\text { Mean points } \\
\text { of passbands } \\
(\AA)\end{array}$ & $\begin{array}{l}\text { Half-power } \\
\text { points } \\
\text { of passbands } \\
(\AA)\end{array}$ & $\begin{array}{l}\text { Number of } \\
\text { measurements }\end{array}$ & $\begin{array}{l}\text { Mean } \\
\text { circular } \\
\text { polarization } \\
(\%)\end{array}$ & $\begin{array}{l}\text { Standard } \\
\text { deviation } \\
(\%)\end{array}$ \\
\hline 3700 & & & -0.063 & \pm 0.086 \\
4400 & 3400,3950 & 20 & +0.015 & 0.052 \\
5400 & 3850,4850 & 20 & +0.041 & 0.033 \\
5500 & 3600,6700 & 10 & +0.052 & 0.034 \\
6300 & 5000,5900 & 20 & +0.121 & 0.032 \\
6900 & 5150,7050 & 16 & +0.076 & \pm 0.040 \\
& 6100,7350 & 20 & &
\end{tabular}


Consequently, we suggest the existence of a small circular polarization increasing towards the longer wavelengths. The wavelength dependence of the polarization is unlike that of interstellar circular polarization (see, e.g., Kemp and Wolstencroft, 1972). It is necessary to confirm the suggestion with an instrument which is capable of measuring the polarization as accurately as $0.01 \%$. A confirmation would be important to the study of X-ray binaries.

\section{References}

Gehrels, T.: 1972, Astrophys. J. 173, L23.

Kemp, J. C. and Wolstencroft, R. D.: 1972, Astrophys. J. 176, L115.

Shulov, O. S. and Kopatskaya, E. N.: 1974, Astrofizika (U.S.S.R.) 10, 120. 